\title{
Teratoma presenting with trichoptysis
}

\author{
Alessandro Marchioni MD, Giulio Rossi MD
}

\section{CASE PRESENTATION}

$\mathrm{A}^{23}$ 23-year-old woman, never smoker and native of Morocco was admitted for recurrent cough accompanied by persistent mucous expectoration containing dark hairs, a phenomenon also known as trichoptysis. Her medical history was unremarkable. Chest x-rays, magnetic resonance imaging and computed tomography $(\mathrm{CT})$ revealed a mass in the anterosuperior mediastinum measuring $7.5 \mathrm{~cm} \times 7.0 \mathrm{~cm} \times$ $6.5 \mathrm{~cm}$, containing fat-density material and air spaces revealing a direct communication with bronchial structures (Figures $1 \mathrm{~A}$ and 1B). Gynecological examination and total-body CT scan ruled out the presence of uterine or ovarian masses. Routine laboratory tests and serum tumour markers including beta-human chorionic gonadotropin were within normal range. The patient underwent bronchoscopy examination of the anterior segment of the left upper bronchus revealing several black-pigmented hairs in the bronchial lumen admixed with whitish mucus (Figure $1 \mathrm{C}$ ). A diagnosis of intrathoracic teratoma of the mediastinum was suggested. The patient refused surgery, but she presented three years later with profuse trichoptysis. The surgical resection of the mass confirmed the clinical diagnosis of mature teratoma.

\section{DISCUSSION}

Teratoma of the lung is a very rare occurrence. Symptoms are generally nonspecific or due to thoracic discomfort related to the physical encumbrance of the mass compressing neighbouring structures. Trichoptysis is secondary to the passage of intratumoural hairs into the bronchial tree through a fistula and occurs in only $10 \%$ to $20 \%$ of intrapulmonary teratomas $(1,2)$. This finding is almost pathognomonic for teratoma, particularly in young women. On CT, teratoma is a heterogeneous, well-demarcated mass with a variably thick fibrous, enhancing wall. The tumour always contains high signal intensity soft tissue (on T1-weighted images), also showing a fluid-rich multi/ unilocular cystic appearance with fat and calcifications (3).

More than $90 \%$ of pulmonary teratomas are benign tumours; complete resection is usually curative and always indicated because malignant transformation may develop.

DISCLOSURES: The authors have no financial disclosures or conflicts of interest to declare.

INFORMED CONSENT: The patient gave informed consent for publication.

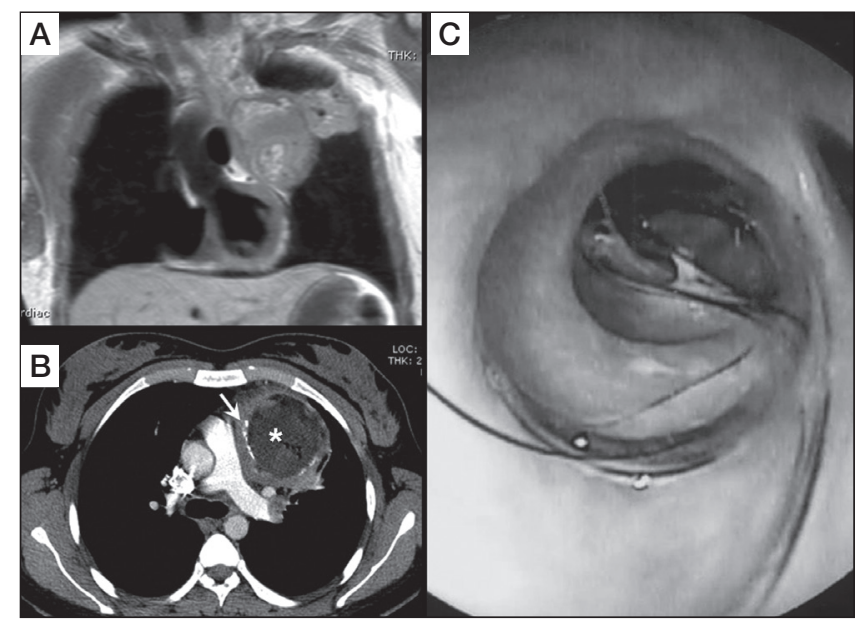

Figure 1) A Magnetic resonance imaging showing a mass with a highsignal intensity unilocular cyst with adipose tissue-like density with direct communication with the anterior segment of the left upper lobar bronchus. B Contrast-enhanced computed tomography scan showing a heterogeneous mass with areas of fat (asterisk), a shell-like calcified wall (arrow) and fluid attenuation. C Bronchoscopy revealing numerous black hairs with dense secretions into the bronchial lumen

\section{KEY LEARNING POINTS}

- Thoracic teratoma is an exceedingly uncommon benign tumour usually occurring in the anterior mediastinum of young adults, with a slight prevalence in females.

- Trichoptysis, due to fistulization between the mass containing hairs and the bronchial tree, is almost diagnostic and may be the first sign of thoracic teratoma.

\section{REFERENCES}

1. Agarwal R, Srivinas R, Saxena AK. Trichoptysis due to an intrapulmonary teratoma. Respir Care 2007;52:1779-81.

2. Rana SS, Swami N, Metha S, Singh J, Biswal S. Intrapulmonary teratoma: an exceptional disease. Ann Thorac Surg 2007;83:1194-6.

3. Moeller KH, Rosado-de-Christenson ML, Templeton PA.

Mediastinal mature teratoma: Imaging features.

Am J Roentgenol 1997;169:985-90.

The 'Images in Respiratory Medicine' section of the Canadian Respiratory Journal aims to highlight the importance of visual interpretation, whether physiological, radiological, bronchoscopic, surgical/thorascopic or histological, in the diagnosis of chest diseases. Submissions should exemplify a classic, particularly dramatic or intriguing presentation of a disease while offering an important educational message to the reader (insightful diagnostic pearls or differential diagnosis, etc). This section is not intended to be a vehicle for publication of case reports (see the Clinico-Pathologic Conferences for case-based leaning series).

Azienda Ospedaliero-Universitaria Policlinico of Modena, Modena, Italy

Correspondence: Dr Alessandro Marchioni, Respiratory Diseases Clinic, Azienda Ospedaliero-Universitaria Policlinico, via del Pozzo, 71-41124, Modena, Italy. Telephone 39-059-4225859, fax 39-059-4222571, e-mail marchioni.alessandro@unimore.it 


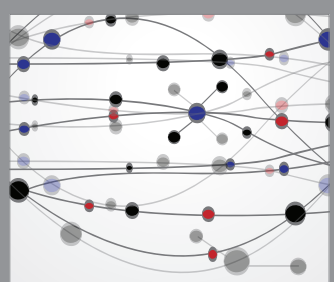

The Scientific World Journal
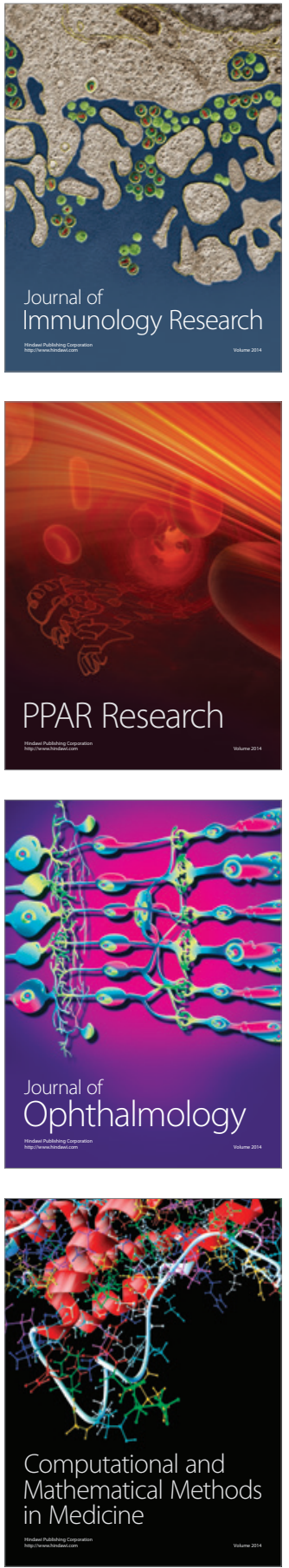

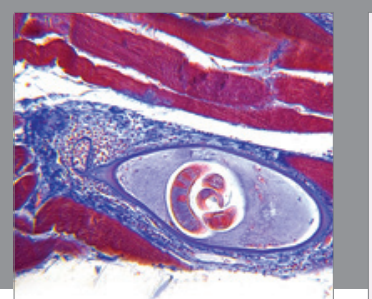

Gastroenterology Research and Practice

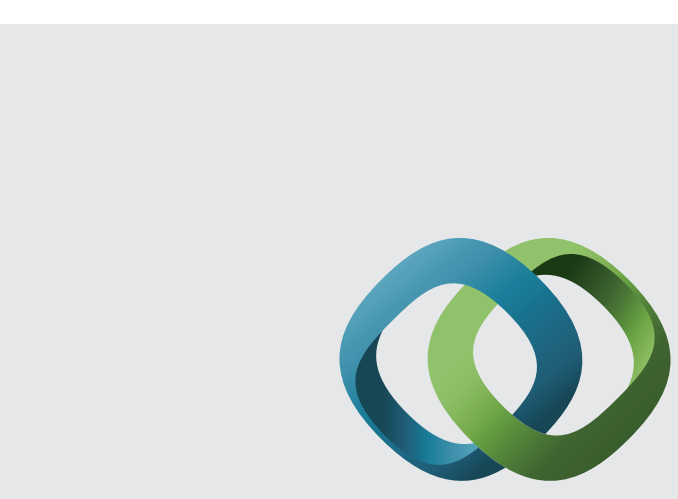

\section{Hindawi}

Submit your manuscripts at

http://www.hindawi.com
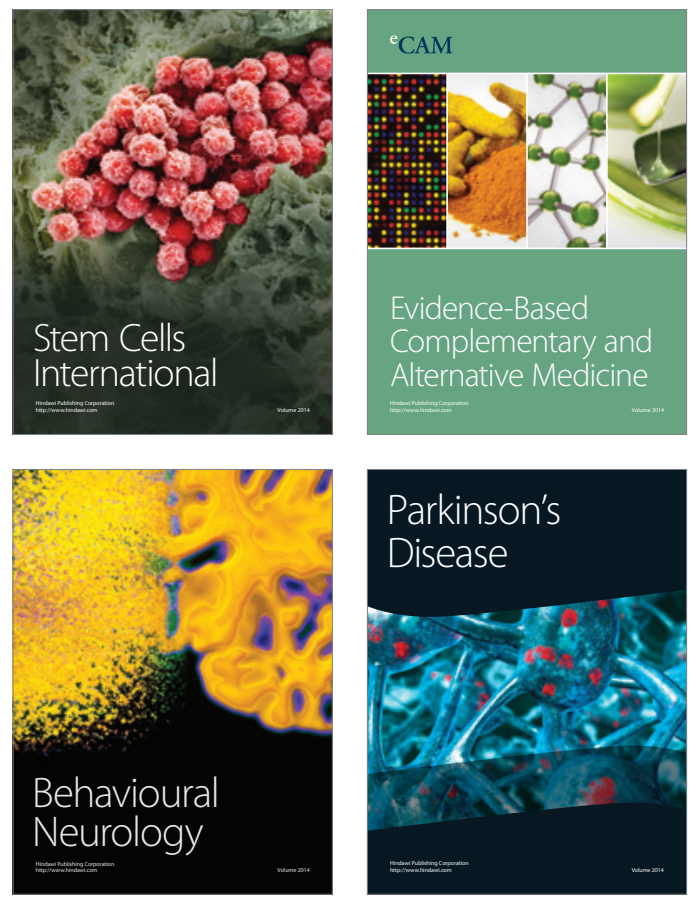
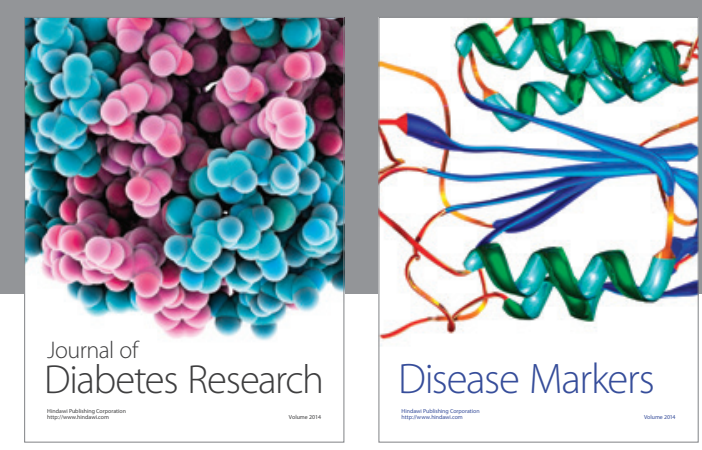

Disease Markers
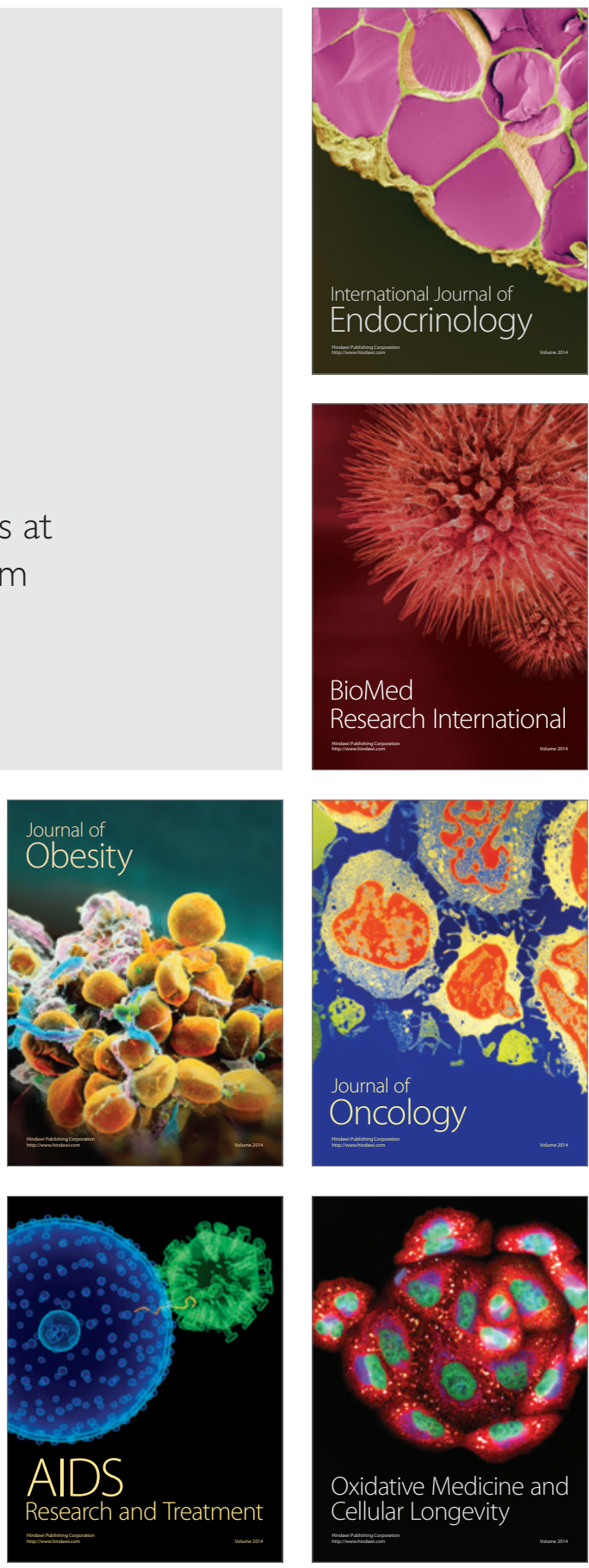